\title{
Primary meningococcal arthritis associated with adult respiratory distress syndrome
}

\author{
A Samanta, A Turner, S Roy, A O'Leary
}

\begin{abstract}
A previously fit and well 20 year old man with primary meningococcal arthritis of the left knee is described. Despite intensive search there was no evidence of extra-articular meningococcal infection. He subsequently developed adult respiratory distress syndrome as a rare and previously undescribed complication.
\end{abstract}

Arthritis which occurs as a secondary complication of meningococcal disease is well recognised and occurs in up to $2-10 \%$ of reported series. Primary meningococcal arthritis occurring in the absence of extra-articular infection is very rare, however. Between 1897 and 1979 only 25 cases of primary meningococcal arthritis were reported, ${ }^{2}$ and subsequently there have been another six. ${ }^{3}$ We report a previously fit young man with primary meningococcal arthritis, who developed an unusual complication of adult respiratory distress syndrome. To our knowledge this has not been described before.

\section{Case report}

A previously fit and well 20 year old man was admitted to the orthopaedic services with a five hour history of pain and swelling in his left knee. On examination he had a fever $\left(39 \cdot 6^{\circ} \mathrm{C}\right)$ with a tachycardia of $120 / \mathrm{min}$. The left knee was hot, swollen, and tender with painful limited movements. Systemic examination was entirely normal. Investigations showed a leucocytosis of $19.5 \times 10^{9} / 1$ (normal $\left.4-11 \times 10^{9} / 1\right)$ with $88 \%$ neutrophils. Urea, creatinine, electrolytes, $x$ ray of the knee, chest $x$ ray, and electrocardiogram were normal. He underwent an arthrotomy with lavage of the left knee, under general anaesthesia, and $50 \mathrm{ml}$ of purulent fluid was withdrawn. After the operation he recovered uneventfully, and treatment was started empirically with intravenous cephalosporins and metronidazole.

About 12 hours later he became acutely short of breath and cyanosed. He had bilateral crepitations and rhonchi. Arterial blood gases showed a $\mathrm{pH} 7 \cdot 27, \mathrm{PCO}_{2} 6 \mathrm{kPa}$, and $\mathrm{Po}_{2} 7 \cdot 6 \mathrm{kPa}$ with a saturation of $84 \%$. Chest $x$ ray showed bilateral patchy opacities (figure A). Blood pressure was $120 / 80 \mathrm{mmHg}$. He was transferred to the intensive therapy unit, where a Swan Ganz catheter was inserted. Pulmonary artery pressure was $25 / 16 \mathrm{mmHg}$ and pulmonary wedge pressure $8 \mathrm{mmHg}$. A diagnosis of adult respiratory distress syndrome was made, and he was given assisted ventilation with positive end expiratory pressure. Microbiological examination of fluid from his knee identified 'meningococcus sensitive to benzylpenicillin' (subsequently confirmed as Neisseria meningitidis group C subtype P1.2), and treatment was started with high dose intravenous benzylpenicillin. His clinical recovery continued very satisfactorily and he was extubated about 24 hours later. Arterial blood gases, urea, and electrolytes were normal, and a chest $x$ ray showed clearing of the previous opacities (figure B).

He was subsequently mobilised on a general ward and antibiotics continued. General clinical examination on numerous occasions was normal. Multiple blood and urine cultures (including initial cultures before starting antibiotic treatment) and swabs from various bodily sources did not grow any organisms. Serum complement and immunoglobulin concentrations were normal. He was discharged from hospital three weeks later, having made a complete recovery.

\section{Discussion}

Arthritis as a complication of meningococcal infection has been described. ${ }^{1}$ It usually occurs in the presence of reported meningococcaemia (either acute or chronic) or with isolation of the organism from other sources such as the throat, with additional features of meningococcal infection, such as fever, variable skin lesions, meningitis, or systemic involvement. ${ }^{2} 3$ The arthritis may be monoarticular, oligoarticular, or polyarticular, ${ }^{45}$ either septic ${ }^{56}$ or sterile, ${ }^{4}$ or associated with tenosynovitis. ${ }^{7}$ Occasionally a 'reactive' postinfective arthritis may be seen. This tends to appear after meningococcal infection and is thought to be immunologically mediated. ${ }^{8-10}$

Primary meningococcal arthritis, however, which is meningococcal infection primarily affecting a joint, in the absence of proved extraarticular infection, is extremely uncommon.

The case described is one of primary meningococcal arthritis as $N$ meningitides was grown only from the affected knee joint and was not cultured from any other site despite intensive efforts. Furthermore, there were no other clinical features of meningococcal disease. In addition, the patient developed clinical and radiological features of adult respiratory distress syndrome shortly after arthrotomy and lavage, and required assisted ventilation.

Acute pulmonary oedema has been described as part of the meningococcal shock syndrome in children and is a potentially life threatening complication. ${ }^{11}$ It is thought that this is due to an increase in pulmonary capillary permeability because of sudden bacteraemia. " 
Chest $x$ ray during $(A)$ and after $(B)$ the development of adult respiratory distress syndrome.
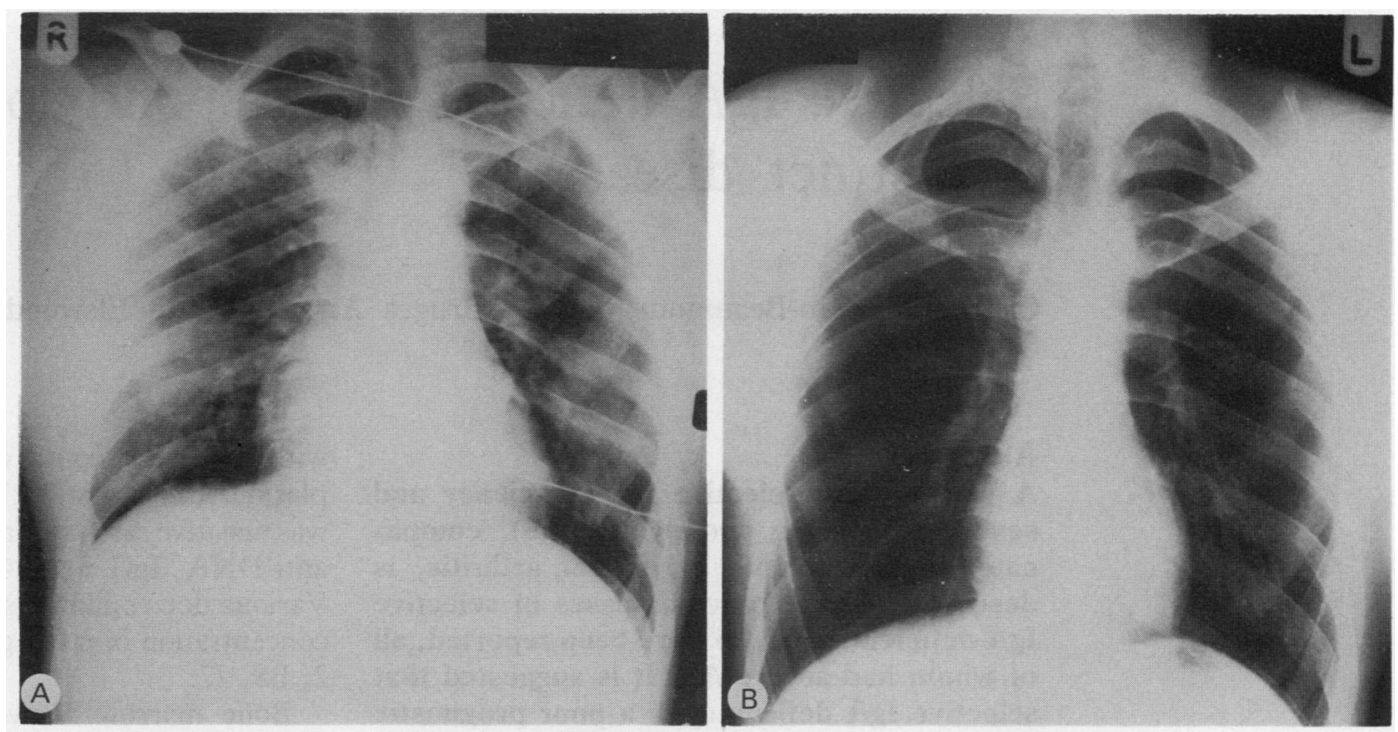

ledge this has not been described before in primary meningococcal arthritis. Meningococcal arthritis and bacteraemia have been described following arthroscopy, ${ }^{12}$ and, possibly, the arthrotomy and lavage performed in our patient induced a transient bacteraemia with 'seeding' in the pulmonary capillary bed.

Antibiotic treatment, which was empirically started immediately after the arthrotomy, might have accounted for the negative cultures at the time of adult respiratory distress syndrome (although initial bacteriological cultures were also negative). Antibiotics might also have contributed to the rapid recovery from a complication which otherwise carries a high mortality. ${ }^{11}$ We emphasise that a septic monoarthritis may occur as the only manifestation of meningoccocal infection, and this may be associated with life threatening systemic complications.

We thank Ms Judy Noble for her secretarial assistance in preparing this manuscript.
1 Pinals R S, Ropes M W. Meningococcal arthritis. Arthritis Rheum 1964; 7: 241-58.

2 Schaad U B. Arthritis in disease due to Neisseria meningitides. Rev Infect Dis 1980; 2: 880-8.

3 Andersson S, Krook A. Primary meningococcal arthritis. Scand F Infect Dis 1987; 19: 51-4.

4 Kidd B L, Hart H H, Grigor R R. Clinical features of meningococcal arthritis: a report of four cases. Ann Rheum Dis 1985; 44: 790-2.

5 Vidal F, Panisello J More on unusual forms of meningococcal disease: septic polyarthritis. Am 7 Med 1986; 81: 561-2.

6 Rosen M, Myers A R, Dickey B. Meningococcaemia presenting as septic arthritis, periconditis and tenosynovitis. Arthritis Rheum 1985; 28: 576-8.

7 Pollet S M, Leek J C. Tenosynovitis in meningococcaemia. Arthritis Rheum 1987; 30: 232-3.

8 Davis J A S, Peters N, Mohammed I, Major G A C, Holborow E J. Circulating immune complexes in a patient wolborow E J. Circulating immune complexes in a patie

9 Greenwood B M, Onyewotu I, Whittle H C. Complement and meningococcal infection. Br Med f 1976; i: 797-9.

10 Larson H E, Nicholson K G, Loewi G, Tyrrell D A J, Posner J. Arthritis after meningococcal infection. $\mathrm{Br} \mathrm{Med} \mathcal{J}$ 1977; i: 618.

11 Kahn A, Brachet E, Blum D. Mecanismes d'apparition de l'oedeme pulmonaire aigu lors due choc meningococcique de l'enfant. Semaine des Hopitaux de Paris 1981; 57: 1925-9.

12 Christopher G W, Jurik K A, Janecki C J, Haake P W, Riley G J, Chessin L N. Meningococcal arthritis, bacteraemia and osteomyelitis following arthroscopy. Clin Orthop 1982; 171: 